\title{
Reduced Expression of A-Type Potassium Channels in Primary Sensory Neurons Induces Mechanical Hypersensitivity
}

\author{
Li-Ying Chien, ${ }^{1 \star}$ Jen-Kun Cheng, ${ }^{2,5 *}$ Dachen Chu, ${ }^{3}$ Chau-Fu Cheng, ${ }^{1}$ and Meei-Ling Tsaur ${ }^{1,4}$ \\ ${ }^{1}$ Institute of Neuroscience, National Yang-Ming University, Taipei, Taiwan, ${ }^{2}$ Department of Anesthesiology, Mackay Memorial Hospital, Taipei, Taiwan, \\ ${ }^{3}$ Department of Neurosurgery in Hoping Branch and ${ }^{4}$ Department of Education and Research, Taipei City Hospital, Taipei, Taiwan, and ${ }^{5}$ Department of \\ Anesthesiology, Taipei Medical University, Taipei, Taiwan
}

\begin{abstract}
A-type $\mathrm{K}^{+}$channels (A-channels) are crucial in controlling neuronal excitability, and their downregulation in pain-sensing neurons may increase pain sensation. To test this hypothesis, we first characterized the expression of two A-channels, Kv3.4 and Kv4.3, in rat dorsal root ganglion (DRG) neurons. Kv3.4 was expressed mainly in the nociceptive DRG neurons, in their somata, axons, and nerve terminals innervating the dorsal horn of spinal cord. In contrast, Kv4.3 appeared selectively in the somata of a subset of nonpeptidergic nociceptive DRG neurons. Most Kv4.3(+) DRG neurons also expressed Kv3.4. In a neuropathic pain model induced by spinal nerve ligation in rats, the protein levels of Kv3.4 and Kv4.3 in the DRG neurons were greatly reduced. After Kv3.4 or Kv4.3 expression in lumbar DRG neurons was suppressed by intrathecal injections of antisense oligodeoxynucleotides, mechanical but not thermal hypersensitivity developed. Together, our data suggest that reduced expression of A-channels in pain-sensing neurons may induce mechanical hypersensitivity, a major symptom of neuropathic pain.
\end{abstract}

Key words: antisense oligodeoxynucleotides; dorsal root ganglion neurons; intrathecal injection; neuropathic pain; potassium channels; spinal nerve ligation

\section{Introduction}

Small- and medium-sized neurons situated in the dorsal root ganglia (DRGs) are primary sensory neurons responsible for pain sensation, also known as nociceptors. Most nociceptors are polymodal and therefore respond to mechanical (touch and pressure), thermal (cold and heat), and chemical stimuli (Julius and Basbaum, 2001; Bear et al., 2007). Nociceptive DRG neurons extend two distinct axons: the central afferents terminate in the superficial dorsal horn of the spinal cord, whereas the peripheral axons innervate skin, muscle, internal organs, etc. There are two types of nociceptors: one with lightly myelinated $\mathrm{A} \delta$ fibers and the other with unmyelinated C-fibers, which can be further divided into peptidergic and nonpeptidergic groups (Hunt and Mantyh, 2001; Costigan et al., 2006). Neuropathic pain, a type of chronic pain, usually results from nerve injury of nociceptors. Mechanical and thermal hypersensitivities are the two major symptoms of neuropathic pain. Because mechanisms underlying

Received Feb. 13, 2007; revised July 12, 2007; accepted July 13, 2007.

This work was supported by National Science Council Grant NSC 95-2320-B-010-032, Mackay Memorial Hospital Grants MMH 9652 and 9679, and Taipei City Hospital Grant 95003-62-134. We thank Dr. Josephine Lai (University of Arizona Health Sciences (enter, Tucson, AZ), Dr. Fu-Chin Liu, Dr. Chou Po Hung, Tien-Chi Hsu, and Jia-Rung Yang for assistance.

*L.-Y.C. and J.-K.C. contributed equally to this work.

Correspondence should be addressed to Meei-Ling Tsaur, Institute of Neuroscience, National Yang-Ming University, 155 Li-Nong Street, Sec 2, Taipei, Taiwan. E-mail: mltsaur@ym.edu.tw.

DOI:10.1523/JNEUROSCI.0604-07.2007

Copyright $\odot 2007$ Society for Neuroscience $\quad$ 0270-6474/07/279855-11\$15.00/0 the symptoms remain unclear, effective treatment is still lacking (Rice and Hill, 2006).

$\mathrm{K}^{+}$channels are crucial in controlling neuronal excitability, and their downregulation in the nociceptors may increase pain sensation (Ocana et al., 2004). A-type $\mathrm{K}^{+}$channels (A-channels), a group of voltage-gated $\mathrm{K}^{+}(\mathrm{Kv})$ channels, are activated transiently and inactivated rapidly (Hille, 2001). There are five A-channels in mammals: Kv1.4, Kv3.4, Kv4.1, Kv4.2, and Kv4.3. Except for Kv3.4 with high-voltage activation, the other four are activated at low voltages (Coetzee et al., 1999). Three different A-type $\mathrm{K}^{+}$currents $\left(I_{\mathrm{A}} \mathrm{s}\right)$ have been detected from rat DRG neurons: two activated at low threshold and one at high threshold (Gold et al., 1996). Because Kv1.4 and Kv4.3 have been identified (Rasband et al., 2001; Huang et al., 2005), it is likely that Kv3.4 underlies the high-voltage-activated $I_{\mathrm{A}}$. Kv1.4 proteins in the somata of DRG neurons are greatly reduced in a neuropathic pain animal model (Rasband et al., 2001). Whether the expression levels of Kv3.4 and Kv4.3 are also affected remains unknown.

In this report, we demonstrated that $\mathrm{Kv} 3.4$ was indeed present in the DRG neurons. Kv3.4(+) DRG neurons were then characterized and compared with those expressing Kv4.3. Applying a model that induces neuropathic pain in rats (Kim and Chung, 1992), we observed a reduction of Kv3.4 and Kv4.3 proteins in the DRG neurons. Although downregulation of A-channels in the nociceptors and neuropathic pain are closely related, it is unclear which occurs first. Our working hypothesis is that if an A-channel is directly involved in pain modulation, suppressing its expres- 
sion in the nociceptors should result in an increase of pain sensation. Interestingly, after suppressing A-channel expression in the DRG neurons by intrathecal injections of antisense oligodeoxynucleotides (ODNs), we found a selective induction of mechanical hypersensitivity in animals.

\section{Materials and Methods}

Animals. Adult male Sprague Dawley rats (250-300 g on the day of surgery) were provided by the Animal Center of National YangMing University. National guidelines of animal care and handling were followed. Rats were housed individually in plastic cages with soft bedding at room temperature and maintained on a $12 \mathrm{~h}$ light/dark cycle with free access to food and water. All of the experiments were approved by the local ethics committee. Behavioral tests for pain assessment were performed in accordance with policies and recommendations of the International Association for the Study of Pain.

L5/L6 spinal nerve ligation. The method of Kim and Chung (1992) was used. Rats were anesthetized with inhalational isoflurane and placed under a microsurgical apparatus in a prone position. To perform unilateral spinal nerve ligation in the right side, a midline incision was made along the back, and the right paraspinal muscles were separated from the spinous processes at the lumbar L4 to sacral S2 levels. After the L6 transverse process was removed and the L4 and L5 spinal nerves were identified, the L5 spinal nerve was tightly ligated with a 6-0 silk thread. The L6 spinal nerve located caudal and medial to the sacroiliac junction was ligated as well. Sham control consisted of rats receiving the same operation without nerve ligation. Rats were allowed to recover after the closure of incision. Animals with motor dysfunction after surgery were excluded from additional experimental procedures. Seven days later, rats displaying normal grooming, ambulation, and weight gain were killed after behavioral tests.

Intrathecal catheterization. The method of Yaksh and Rudy (1976) was used. After a rat was anesthetized with inhalational isoflurane, the occipital magnus in the dorsal side of neck was opened and the dura mater was incised. A catheter ( $\mathrm{PE}-5$ polyethylene tubing) was inserted intrathecally and advanced caudally $8.5 \mathrm{~cm}$, and the neck incision was closed with sutures. Five days later, rats displaying normal grooming, ambulation, and weight gain were used for intrathecal injection. Catheter placements were verified by visual inspection after the animal was killed.

Intrathecal injection of ODNs. We used the Kv3.4 antisense ODN sequence $5^{\prime}$-CCTCCTTCAGACATGTTTTGGACG-3', complementary to nucleotides 59-82 of rat Kv3.4 coding region (Schroter et al., 1991) (GenBank accession number X62841). The related Kv3.4 mismatch ODN sequence was $5^{\prime}$-CCTGGTTCACAGATCTTTTCCACG-3', with seven different bases underlined. Kv4.3 antisense ODN sequence was 5'-TCATCTTGCCGCTTGTTCTTGTCG-3', complementary to nucleotides 96-119 of the rat Kv4.3 coding region (Tsaur et al., 1997) (GenBank accession number L48619). The related Kv4.3 mismatch ODN sequence was 5'-TCATGTTCGCGGTTCTTGTTCTCG-3', with seven different bases underlined. After fluorophor tetramethyl-6carboxyrhodamine was conjugated to the $5^{\prime}$ end of ODN, the fluorescence-tagged ODN was purified by PAGE. All ODNs were dissolved in water to a concentration of $9 \mu \mathrm{g} / \mu \mathrm{l}$. For each intrathecal injection, $5 \mu \mathrm{l}$ of ODNs was used followed by a $15 \mu \mathrm{l}$ normal saline flush.

Behavioral tests. Two types of tests (mechanical and thermal sensitivities) were performed $1 \mathrm{~d}$ before spinal nerve ligation or intrathecal ODN injection to get the baseline values. Tests were performed once daily at $2-4 \mathrm{pm}$ by an examiner blinded to the treatment groups. For rats with spinal nerve ligation, tests were performed only on the hindpaw of the ligated side. For rats with intrathecal ODN injection, tests were performed on both hindpaws to obtain mean values.

Mechanical sensitivity of rat hindpaw was assessed by von Frey filaments (Stoelting, Wood Dale, IL). Rats were placed in a transparent plastic dome with a metal-mesh floor allowing access to the plantar surface of hindpaws, with a habituation time $\sim 30 \mathrm{~min}$ before testing. The filament was pressed perpendicular to the plantar surface of the hindpaw with sufficient force to cause a slight buckling for $6 \mathrm{~s}$. A positive response was noted when the hindpaw was sharply withdrawn. Flinching immediately after the removal of the filament was also considered as a positive response. The force (in grams) producing a 50\% likelihood of withdrawal was determined by the "up-down" method (Chaplan et al., 1994). Each trial was repeated twice at $2 \mathrm{~min}$ intervals, and the mean value represented the paw withdrawal threshold.

Thermal sensitivity of rat hindpaw was measured by an Analgesia Meter apparatus (IITC/Life Science Instruments, Woodland Hills, CA) as described by Hargreaves et al. (1988). Rats were placed on a temperature-controlled, 3-mm-thick glass floor and habituated for 30 min before testing. A movable light box was located beneath the glass floor, and radiant heat was focused on the planter surface of the hindpaw. A cutoff time was set at $20 \mathrm{~s}$ to avoid tissue damage. Light intensity was adjusted to obtain a baseline value within 10-12 s. Ten withdrawal latencies were collected with at least 5 min intervals and the middle six latencies were averaged.

Perfusion. After deep anesthesia by intraperitoneal injection of sodium pentobarbital $(100 \mathrm{mg} / \mathrm{kg})$, rats were perfused transcardially with normal saline followed by $4 \%$ paraformaldehyde in $0.1 \mathrm{M}$ phosphate buffer $(0.08$ $\mathrm{M} \mathrm{K}_{2} \mathrm{HPO}_{4}, 0.02 \mathrm{M} \mathrm{NaH}_{2} \mathrm{PO}_{4}, \mathrm{pH}$ 7.4) for $20 \mathrm{~min}$. DRGs, dorsal root fibers, sciatic nerves, and the lumbar segment of the spinal cord were removed and postfixed in the same fixative for $30 \mathrm{~min}$ (except $60 \mathrm{~min}$ for spinal cord) at room temperature. All specimens were cryoprotected in $30 \%(\mathrm{w} / \mathrm{v})$ sucrose in $0.1 \mathrm{M}$ phosphate buffer.

Antibody specificity. The specificity of rabbit anti-Kv3.4 (APC-019; Alomone Labs, Jerusalem, Israel) has been described previously (Brooke et al., 2004). Using brain tissues of Kv4.3 knock-out mice, the specificity of mouse anti-Kv4.3 (K75/41; NeuroMab Facility, University of Califor- 


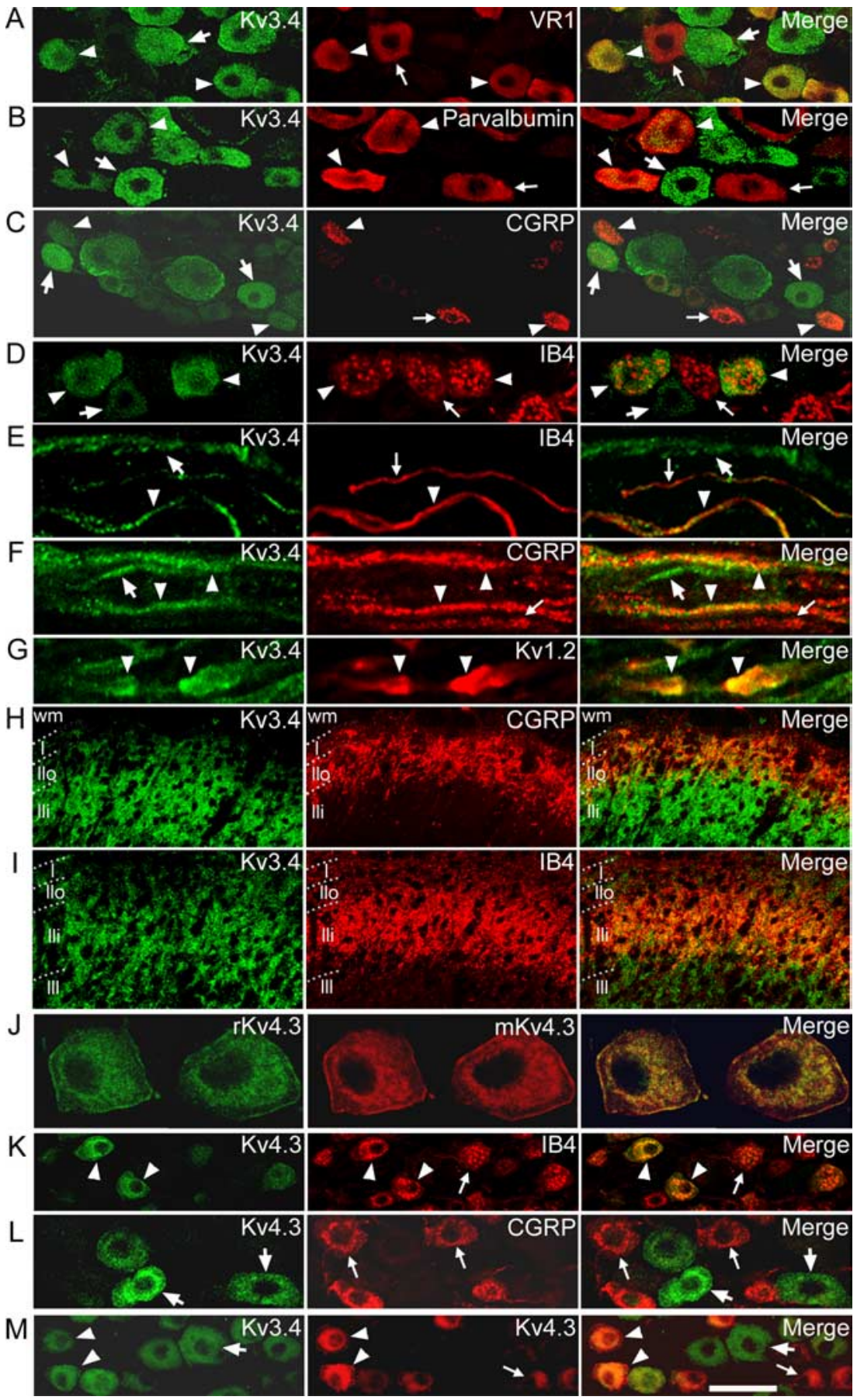

Figure 2. Characterization of Kv3.4(+) and Kv4.3(+) DRG neurons. Double immunofluorescent staining was performed for confocal images. The arrowhead indicates the colocalization of Kv subunit and marker; the arrow with a large arrowhead indicates a cell (or fiber) expressing Kv subunit but not marker; and the arrow with a small arrowhead indicates a cell (or fiber) expressing marker but not Kv subunit. $\boldsymbol{A}-\boldsymbol{D}$, Colocalization of Kv3.4 with VR1 (A), parvalbumin (B), CGRP ( $($ ), and IB4 (D) in the cell bodies of some DRG neurons. Note the small- to medium-sized neurons shown in $C$, Kv3.4-IR is stronger in Kv3.4(+)/CGRP(-) than Kv3.4(+)/CGRP( + ) neurons. $\boldsymbol{E}-\boldsymbol{G}$, Within the sciatic nerve, Kv3.4 appears in the IB4 $(+)$ fibers $(\boldsymbol{E}), \operatorname{CGRP}(+)$ fibers $(\boldsymbol{F})$, and Kv1.2(+) juxtaparanodes (G). $\boldsymbol{H}, \boldsymbol{I}$, In the dorsal horn, Kv3.4 is colocalized with CGRP in laminas I and $\|_{0}(\boldsymbol{H})$ and with IB4 in the dorsal and medial parts of lamina lli $(\boldsymbol{I})$.J, Both the rabbit anti-Kv4.3 (rKv4.3) and the mouse anti-Kv4.3 (mKv4.3) antibodies label the same subset of DRG neurons, with stronger immunoreactivity in the perinuclear Golgi complex than somatic surface. $\boldsymbol{K}, \boldsymbol{L}, A$ All Kv4.3( + ) neurons bind IB4 $(\boldsymbol{K})$, but none express $(\mathrm{GRP}(\boldsymbol{L}) . \boldsymbol{M}, \mathrm{Kv3} .4$ and $\mathrm{Kv4}$.3 are coexpressed in a subset of DRG neurons. Scale bar: (in $\boldsymbol{M}) \boldsymbol{A}, \boldsymbol{B}, 53 \mu \mathrm{m} ; \boldsymbol{C}, 66 \mu \mathrm{m} ; \boldsymbol{D}, \boldsymbol{E}, 23 \mu \mathrm{m} ; \boldsymbol{F}, \boldsymbol{G}, 12 \mu \mathrm{m} ; \boldsymbol{H}, \boldsymbol{I}, 66 \mu \mathrm{m} ; \boldsymbol{J}, 11 \mu \mathrm{m} ; \boldsymbol{K}, 56 \mu \mathrm{m} ; \boldsymbol{L}, 32 \mu \mathrm{m} ; \boldsymbol{M}, 62 \mu \mathrm{m}$.

nia Davis, Davis, CA) has been established (Burkhalter et al., 2006). In addition to Western blot and immunoprecipitation analysis (Hsu et al., 2003), rabbit anti-Kv4.3 (APC-017; Alomone Labs, the AN01-04 batches) has been verified to specifically detect Kv4.3 in rat cerebellum, spinal cord, and DRG (Hsu et al., 2003; Huang et al., $2005,2006)$. Here, identical patterns were found in the spinal cord and DRG sections immunostained with NeuroMab anti-Kv4.3 and Alomone Labs antiKv4.3 (the AN01-04 but not AN05-07 batches) (supplemental data, available at www.jneurosci.org as supplemental material) (see Fig. 1). Although nonspecific staining of Alomone Labs anti-Kv4.3 has been reported previously (Burkhalter et al., 2006) the potential for such nonspecificity should be limited to the AN05, AN06, and AN07 batches.

Single antigen immunohistochemistry. DRGs in random orientation were cut with a cryostat into 20 $\mu \mathrm{m}$ sections and mounted directly onto gelatincoated slides. The sciatic nerve and the dorsal root were then cut along the parasagittal orientation into $10 \mu \mathrm{m}$ strands, and fibers were teased in Trisbuffered saline (TBS) (25 mm Tris, pH 7.5, 0.85\% $\mathrm{NaCl}$ ) before attachment to slides. Spinal cord sections $(20 \mu \mathrm{m})$ in the transverse orientation were processed in a floating manner. After washing in TBS, sections were treated with $0.2 \%$ hydrogen peroxide until no bubbles appeared. Nonspecific binding was blocked by $3 \%$ normal goat or horse serum plus $2 \%$ bovine serum albumin in TBS containing $0.3 \%$ Triton X-100 for $1.5 \mathrm{~h}$. For DRG sections, the following primary antibodies and their dilution factors were used: rabbit anti-Kv3.4 (1:700), rabbit or mouse anti-Kv4.3 (1:500), mouse antineurofilament-M (NF-M) (MAB1621; 1:300 Chemicon, Temecula, CA), and guinea pig anticalcitonin gene-related peptide (CGRP) (T-5027; 1:1000; Bachem, San Carlos, CA). For dorsal root and sciatic nerve fibers, rabbit anti-Kv3.4 (1:100) and rabbit or mouse anti-Kv4.3 (1:50) were used. For spinal cord sections, rabbit anti-Kv3.4 (1:1500) and rabbit or mouse anti-Kv4.3 (1:500) were used After overnight reaction with primary antibody, sections were incubated for $1.5 \mathrm{~h}$ with one of the following biotinylated secondary antibodies: goat antirabbit IgG (1:1000; Pierce, Rockford, IL), horse antimouse IgG (1:500; Vector Laboratories, Burlingame, $\mathrm{CA})$, or goat anti-guinea pig IgG (1:1000; Vector Laboratories). After the incubation of avidin-biotinhorseradish peroxidase complex (1:160; Pierce) for $1.5 \mathrm{~h}$, the antigen was visualized by combining equal volumes of ammonium nickel sulfate $(30 \mathrm{mg} / \mathrm{ml}$ in $0.1 \mathrm{~m}$ sodium acetate, $\mathrm{pH} 6.0$ ) and diaminobenzidine $(4 \mathrm{mg} / \mathrm{ml}$ in TBS) in the presence of $0.01 \%$ hydrogen peroxide. Floating sections of spinal cord were spread flat on slides and air dried. Sections on slides were dehydrated through an ethanol gradient $(70 \%$ once, $95 \%$ twice, and $100 \%$ twice, 1.5 min each) and twice in xylene for $3 \mathrm{~min}$. Sections on slides were coverslipped with mounting medium (Permount; Merck, Darmstadt, Germany). Images were acquired with a DXM1200 digital camera connected to an Eclipse E800 light microscope (Nikon, Melville, $\mathrm{NY}$ ) and processed with PHOTOSHOP 8.0 software (Adobe Systems, Mountain View, CA).

Double fluorescent immunohistochemistry. For double staining using two primary antibodies derived from different animal species, sections were processed similarly as described above (see Single antigen immunohistochemistry), except that treatment with hydrogen peroxide was omitted and sections were incubated simultaneously with two primary antibodies. For DRG sections, the primary antibodies and their dilution factors were rabbit anti-Kv3.4 (1:600), rabbit or mouse anti-Kv4.3 (1:200), guinea pig 
Table 1. Quantitative measurements of colocalization of Kv3.4 and markers in rat lumbar DRG neuron somata

\begin{tabular}{lll}
\hline & \multicolumn{1}{l}{ Number of DRG neurons } & \\
\cline { 2 - 3 } & Marker(+)/Kv3.4(+)\% & Kv3.4(+)/marker(+)\% \\
\hline Marker of nociceptor & 345 of $986(35.0)$ & 345 of $607(56.8)$ \\
$\quad$ GRP & 401 of $801(50.1)$ & 401 of $743(54.0)$ \\
IB4 & 562 of $895(62.8)$ & 562 of $792(71.0)$ \\
VR1 & & \\
$\begin{array}{l}\text { Marker of proprioceptor } \\
\quad \text { Parvalbumin }\end{array}$ & 77 of $509(15.1)$ & 77 of $154(50.0)$ \\
\hline
\end{tabular}

anti-CGRP (1:1000), mouse anti-parvalbumin (PARV-19; 1:1000; Sigma, St. Louis, MO), and goat anti-vallinoid receptor 1 (VR1) (R-18; 1:100; Santa Cruz Biotechnology, Santa Cruz, CA). For sciatic nerve fibers, rabbit antiKv3.4 (1:100) and guinea pig anti-CGRP (1:500) were used. For spinal cord sections, rabbit anti-Kv3.4 (1:150) and guinea pig anti-CGRP (1:800) were used. Secondary antibodies included Alexa fluor 488-conjugated goat or donkey anti-rabbit IgG (1:500; Invitrogen, Eugene, OR), Alexa fluor 594conjugated goat or donkey anti-mouse IgG (1:500; Invitrogen), and rhodamine Red-X-conjugated donkey anti-goat or anti-guinea pig IgG (1:500; Jackson ImmunoResearch, West Grove, PA). For isolectin B4 (IB4) labeling, after washing in $0.1 \mathrm{M}$ Tris buffer containing $1 \mathrm{mM} \mathrm{CaCl}_{2}$ to remove secondary antibody, sections were incubated with Alexa fluor 594-conjugated IB4 (Invitrogen) in the same solution for $2 \mathrm{~h}, 1: 1000$ for spinal cord floating sections and 1:400 for DRG sections on slide. Floating sections were spread flat onto slides for attachment, and sections on slides were mounted with the anti-fading medium Fluoromount-G (Southern Biotech, Birmingham, AL) under coverslips.

For double staining using two primary antibodies derived from the same species (rabbit in our report), the tyramide amplification method was used (Shindler and Roth, 1996; Huang et al., 2005). On the first day, the first primary antibody was applied at a concentration much lower than normally used for double immunofluorescent staining. On the second day, with intervening washes with TBS containing $0.1 \%$ Triton $\mathrm{X}-100$, the signal for the first primary antibody was amplified sequentially by the biotinylated donkey anti-rabbit IgG (1:1000; Jackson ImmunoResearch), avidin-biotin-horseradish peroxidase complex (1:200), biotinylated tyramide (1:1000), and rhodamine Red-X-conjugated streptavidin (1:500; Jackson ImmunoResearch) for $1 \mathrm{~h}$ each. The second primary antibody was then applied overnight at a concentration normally used for double immunofluorescent staining. On the third day, Alexa fluor 488-conjugated donkey anti-rabbit IgG (1:200) was applied to visualize the second primary antibody. For Kv1.2/Kv3.4 double labeling in the sciatic nerve, sections were incubated with rabbit anti-Kv1.2 (1:1000; Alomone Labs) before rabbit anti-Kv3.4 (1:100). For Kv4.3/ Kv3.4 double staining in the DRG, sections were incubated with rabbit anti-Kv4.3 (1:1500) before rabbit anti-Kv3.4 (1:600). Images were collected using an Olympus FV300 confocal laser scanning microscope and processed with Adobe Photoshop 8.0 software.

Quantitative measurements. To obtain the cell profiles of Kv3.4(+) and Kv4.3(+) DRG neurons, three naive rats were killed and only one L5 DRG was used from each rat. In each L5 DRG, three sections were sampled approximately in the middle part of DRG with a distance of every 80 $\mu \mathrm{m}$. After immunostaining, Kv3.4(+) and Kv4.3(+) DRG neurons with obvious nuclear localization at or near the center of cells were measured for diameter and counted. To quantify immunoreactivity (IR) obtained from single antigen immunohistochemistry, immunoreactivity within a DRG neuron (supplemental data, available at www.jneurosci.org as supplemental material) (see Fig. 2) or certain area in the sciatic nerve/dorsal horn was measured by SCION IMAGE BETA 4.02 software (Scion, Frederick, MD). Differences between two experimental groups were compared by Student's $t$ test with statistical significance threshold $p<0.05$.

\section{Results}

$\mathrm{Kv} 3.4$ is expressed mainly in the C-fiber nociceptors

To investigate whether Kv3.4 is expressed in the DRG neurons, we performed immunohistochemistry in rat lumbar DRGs.
Table 2. Quantitative measurements of colocalization of Kv4.3 and markers in rat lumbar DRG neuron somata

\begin{tabular}{lll}
\hline & \multicolumn{2}{l}{ Number of DRG neurons } \\
\cline { 2 - 3 } & Marker(+)/Kv4.3(+)\% & Kv4.3(+)/marker $(+) \%$ \\
\hline $\begin{array}{l}\text { Marker of nociceptor } \\
\text { CGRP }\end{array}$ & 0 of 339 & 0 of 372 \\
IB4 & 241 of $241(100)$ & 241 of $543(44.3)$ \\
VR1 & 287 of $384(74.7)$ & 287 of $573(50.1)$ \\
Marker of proprioceptor & & \\
$\quad$ Parvalbumin & 0 of 271 & 0 of 99 \\
\hline
\end{tabular}

Forty-eight percent of DRG neurons (858 of 1780 of total cell counts) showed Kv3.4-IR in their cell bodies (Fig. $1 A$ ). The somatic sizes of $\mathrm{Kv} 3.4(+)$ neurons were measured and divided into three groups: $42 \%$ small-diameter $(\leq 30 \mu \mathrm{m})$, 34\% mediumdiameter $(\sim 31-40 \mu \mathrm{m})$, and 24\% large-diameter $(>40 \mu \mathrm{m})$ (Fig. $1 F$ ). Similar distributions were detected at the cervical and thoracic levels (data not shown). Small- and medium-sized $\mathrm{Kv3.4(+)}$ neurons showed intense Kv3.4-IR in the cytoplasm. In contrast, faint Kv3.4-IR appeared only on the surfaces of largesized Kv3.4(+) neurons (Fig. $1 A$ ). Various markers were used to characterize $\mathrm{Kv} 3.4(+)$ neurons. VR1 and parvalbumin are markers for the nociceptive and proprioceptive DRG neurons, respectively (Carr et al., 1989; Julius and Basbaum, 2001). Double immunofluorescent staining data revealed that $63 \% \mathrm{Kv} 3.4(+)$ neurons were $\operatorname{VR} 1(+)$ and $15 \%$ were parvalbumin $(+)$ (Fig. $2 A, B$; Table 1$)$, suggesting that the majority of Kv3.4(+) DRG neurons are nociceptors. Peptidergic and nonpeptidergic $\mathrm{C}$-fiber neurons were identified by CGRP and IB4 labeling (overlap, $<4 \%$ ), respectively (Julius and Basbaum, 2001; Zwick et al., 2002). We found that $35 \%$ of $\mathrm{Kv} 3.4(+)$ neurons were $\operatorname{CGRP}(+)$ and $50 \%$ were IB4 $(+)$ (Table 1$)$. Kv3.4-IR was much stronger in $\operatorname{IB} 4(+)$ than CGRP $(+)$ neurons (Fig. $2 C, D)$. Thus, Kv3.4 is expressed mainly in the $\mathrm{C}$-fiber nociceptors, primarily in the nonpeptidergic group.

\section{Kv3.4 proteins also appear in the axons and the central afferent terminals of C-fiber nociceptors}

To examine whether Kv3.4 is also present in the axons and the nerve terminals of DRG neurons, we performed immunostaining of the sciatic nerve (peripheral axons), dorsal root (central axons), and spinal cord (the nerve terminals of central axons). The sciatic nerve, a bundle of axons, projects from the L4-L6 DRG neurons to the hindpaw of the rat. We found Kv3.4-IR in many thin fibers within the sciatic nerve (Figs. $1 B, 4 A$, left panels). Colocalization with IB4 or CGRP indicated that the thin axons were C-fibers (Fig. 2E,F). Less frequently, Kv3.4-IR was seen in some juxtaparanode-like structures (Fig. $1 B$ ). Juxtaparanodes are structures flanking the node of Ranvier along myelinated fibers, and Kv1.2 has been used as a marker (Rasband et al., 2001). Double staining data showed that all Kv3.4(+) structures were $\mathrm{Kv1.2(+)}$ and some Kv1.2(+) juxtaparanodes were Kv3.4(+) (Fig. $2 G$ ), confirming that Kv3.4 proteins are localized in the juxtaparanodes. The distribution of Kv3.4 in the dorsal root was similar to that in the sciatic nerve (Fig. 1C).

In the dorsal horn of spinal cord, punctate Kv3.4(+) structures in laminas I-III have been suggested to be the central afferent terminals of DRG neurons (Brooke et al., 2004). We found stronger Kv3.4-IR in lamina II (Fig. $1 D$ ), especially in the inner region (Fig. $1 E$ ). Lamina II includes the outer (IIo) and inner (IIi) regions (Zylka et al., 2005). IIi can be further divided into the dorsal, medial, and ventral parts (Huang et al., 2005). CGRP $(+)$ 


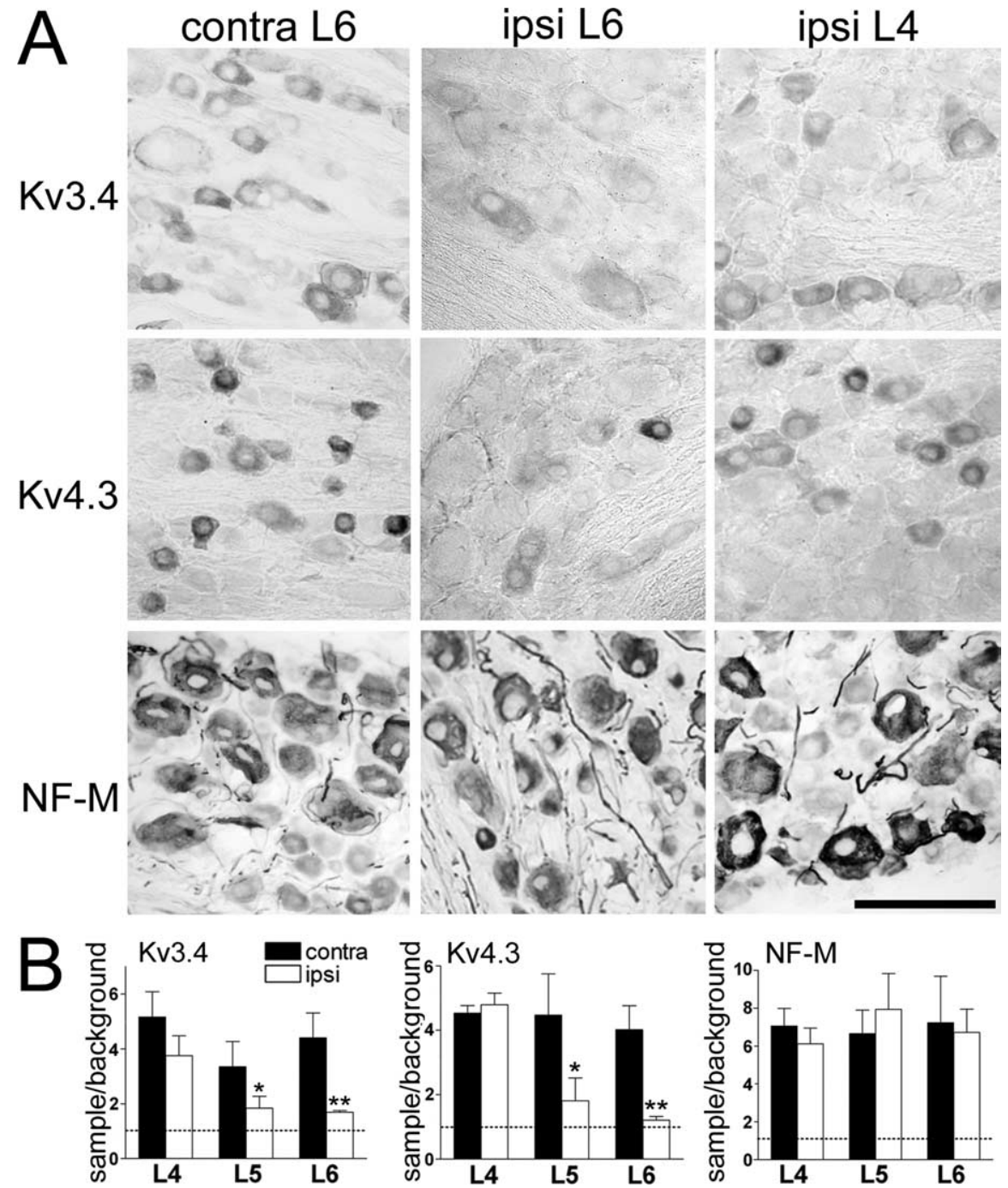

Figure 3. Reduced expression of Kv3.4 and Kv4.3 in the somata of DRG neurons after unilateral L5/L6 spinal nerve ligation. $\boldsymbol{A}$, DRG sections of the nonligated (contra) and ligated (ipsi) sides were immunostained with antibody against Kv3.4, Kv4.3, or NF-M. Compared with the contralateral L5/L6 (only L6 is shown), Kv3.4 and Kv4.3-IR are greatly reduced in the ipsilateral L5/L6 but not L4, whereas NF-M-IR shows no difference. Scale bar, $116 \mu \mathrm{m}$. B, Quantitative data. The value in the $y$-axis is obtained by dividing the sample value with the background value, and the value 1 indicates that the immunoreactivity of the sample is the same as that of background. $n \geq 3$; ${ }^{*} p<0.05,{ }^{* *} p<0.01$, comparing the ipsilateral side with the contralateral side at each lumbar level by Student's $t$ test.

C-fibers target laminas I and IIo, IB4 $(+)$ C-fibers terminate in the dorsal and medial parts of IIi, and PKC $\gamma$ marks in the ventral part of IIi (Fig. 2 H,I) (Zylka et al., 2005). Kv3.4 was colocalized with CGRP in laminas I and IIo (Fig. $2 H$ ), with IB4 in the dorsal and medial parts of IIi (Fig. 2I), but not with PKC $\gamma$ in the ventral part of IIi (data not shown). Together, in addition to the somata, Kv3.4 proteins are also distributed in the axons and the nerve terminals of C-fiber nociceptors.

\section{Kv4.3 is expressed exclusively in a subset of nonpeptidergic nociceptors}

To examine whether other A-channels show expression patterns similar to Kv3.4, we characterized Kv4.3(+) DRG neurons for comparison. Unlike Kv3.4, Kv4.3-IR was completely absent from the sciatic nerve and dorsal root fibers (data not shown). Kv4.3-IR appeared only in the cell bodies of some small-sized DRG neurons, strongly in the perinuclear compartment and weakly on the somatic surface, as revealed by two different Kv4.3 antibodies (Fig. 2J). The intracellular localization of $\mathrm{Kv} 4.3$ was similar to that of Kv4.2 (another A-channel), primarily in the cis-Golgi network and to a lesser extent in the trans-Golgi network (O'Callaghan et al., 2003). It is known that Kv4.2 proteins are segregated in the Golgi complex after biosynthesis, transported to cell surface by $\mathrm{Kv}$ channel interacting protein 1 (KChIP1) via a postendoplasmic reticulum vesicular pathway, and exert ion channel function on plasma membrane (An et al., 2000; Hasdemir et al., 2005). Based on the high similarity between Kv4.2 and Kv4.3 (Birnbaum et al., 2004), it is likely that $\mathrm{Kv} 4.3$ proteins are clustered in the Golgi complex of DRG neurons. Next, the cell profiles of Kv4.3(+) DRG neurons were examined. At the lumbar level, 26\% (403 of 1546 of total cell counts) of the neurons were $\mathrm{Kv} 4.3(+)$ : $57 \%$ small-diameter $(\leq 30 \mu \mathrm{m}), 40 \%$ mediumdiameter $(\sim 31-40 \mu \mathrm{m})$, and 3\% largediameter $(>40 \mu \mathrm{m})$ (Fig. $1 G)$. Similar distributions were detected at the cervical and thoracic levels (data not shown). Interestingly, $100 \%$ of $\mathrm{Kv} 4.3(+)$ neurons bound IB4 and 44\% of IB4 $(+)$ neurons showed Kv4.3-IR (Fig. 2K, Table 2). Kv4.3(+) neurons expressed neither CGRP nor parvalbumin (Fig. $2 L$, Table 2). These results demonstrate that $\mathrm{Kv} 4.3$ is selectively expressed in a subset of nonpeptidergic C-fiber nociceptors. We also analyzed whether Kv3.4 and Kv4.3 were colocalized in the somata of some DRG neurons. Seventy-nine percent (547 of 691 of total cell counts) of Kv4.3(+) neurons showed Kv3.4-IR, and 57\% (547 of 968 of total cell counts) of $\mathrm{Kv} 3.4(+)$ neurons showed Kv4.3-IR (Fig. 2M). These data indicate that Kv3.4 and Kv4.3 are coexpressed in a subset of nonpeptidergic C-fiber nociceptors.

\section{Expression of A-channels in nociceptors is reduced after nerve injury}

To investigate whether the expression levels of Kv3.4 and Kv4.3 in the DRG neurons are altered after nerve injury, we ligated the right side lumbar L5 and L6 spinal nerves of rats. Behavioral tests for mechanical and thermal sensitivities were used to assess the degree of pain in the right hindpaw. Both mechanical and thermal sensitivities increased $7 \mathrm{~d}$ after ligation (mechanical sensitivity, $18.2 \pm 5.8 \mathrm{~g} 1 \mathrm{~d}$ before ligation, $2.0 \pm 0.9 \mathrm{~g} 7 \mathrm{~d}$ after ligation, $n=6, p<0.001$; thermal sensitivity, $11.9 \pm 1.3 \mathrm{~s}$ before ligation, $5.6 \pm 1.0 \mathrm{~s}$ after ligation, $n=6, p<0.001)$. Rats were then killed for immunohistochemical analysis. In the somata of L5 and L6 DRG neurons of the ligated side, Kv3.4-IR was reduced to 55 and $38 \%$ of the nonligated side, and Kv4.3-IR was reduced to 40 and $30 \%$, respectively (Fig. $3 A, B$ ). In contrast, NF-M-IR was not affected (Fig. $3 A, B$ ). In the sciatic nerve, Kv3.4-IR was reduced to $58 \%$ (Fig. $4 A, C$ ). CGRP-IR in small-caliber fibers also decreased, whereas NF-M-IR in medium- and large-caliber fibers showed 
no significant change (Fig. $4 A, C$ ). It is known that DRG neurons become apoptotic $2 \mathrm{~d}$ after spinal nerve crush (Campana and Myers, 2003), and a significant loss of small-sized DRG neurons has been observed 8 weeks after sciatic nerve transection (Tandrup et al., 2000). To examine the extent of apoptosis, we applied a terminal deoxynucleotidyl transferasemediated dUTP nick end labeling assay. Apoptotic nuclei were randomly distributed in the DRG, with or without ligation, in both large- and small-sized neurons (supplemental data, available at www.jneurosci. org as supplemental material) (Fig. 3). Four percent of total L5 DRG neurons were apoptotic in the nonligated side and $9 \%$ on the ligated side $(n=5 ; p<0.05)$, with only a $5 \%$ difference. Compared with the $\sim 50 \%$ reduction of $\mathrm{Kv} 3.4$ or $\mathrm{Kv} 4.3-\mathrm{IR}$ in the L5 DRG neurons, the contribution of ligationinduced apoptosis to overall immunoreactivity reduction was limited. Thus, the immunoreactivity reduction is likely caused by a decrease of A-channel expression.

The protein levels of Kv3.4 and Kv4.3 in the dorsal horn $7 \mathrm{~d}$ after ligation were also quantified. In the L5 dorsal horn of the ligated side, Kv3.4-IR was reduced to $69 \%$, but Kv4.3-IR was not (Fig. 4B,D). $\mathrm{Kv} 4.2$ has been detected from the somatodendritic compartment of certain dorsal horn neurons, although it is completely absent from DRG neurons (Huang et al., 2005). Similar to Kv4.3, Kv4.2-IR in the dorsal horn was unaltered by ligation (data not shown). Because Kv3.4 appears mainly in the axon terminals of DRG neurons projecting to laminas I-III (Fig. $1 E$ ) (Brooke et al., 2004), decrease of Kv3.4-IR in lamina I-III was contributed by reduced expression in the DRG neurons. In contrast, no alteration of Kv4.2 and Kv4.3-IR in lamina II indicates that both channels remain normally expressed in dorsal horn neurons. Thus, $7 \mathrm{~d}$ after spinal nerve ligation, expression of A-channels was greatly reduced in the DRG but not dorsal horn neurons.

Suppressing A-channel expression by antisense ODNs selectively induces mechanical hypersensitivity

Selective knockdown of certain gene expression in the DRG neurons has been achieved by the intrathecal injections of antisense ODNs (Lai et al., 2002; Gold et al., 2003; Bourinet et al., 2005; Son et al., 2007). Gene-specific antisense ODNs hybridize to mRNAs in the cytoplasm to disrupt translation, which results in a decrease of target protein synthesis. To determine the temporal profile of ODN
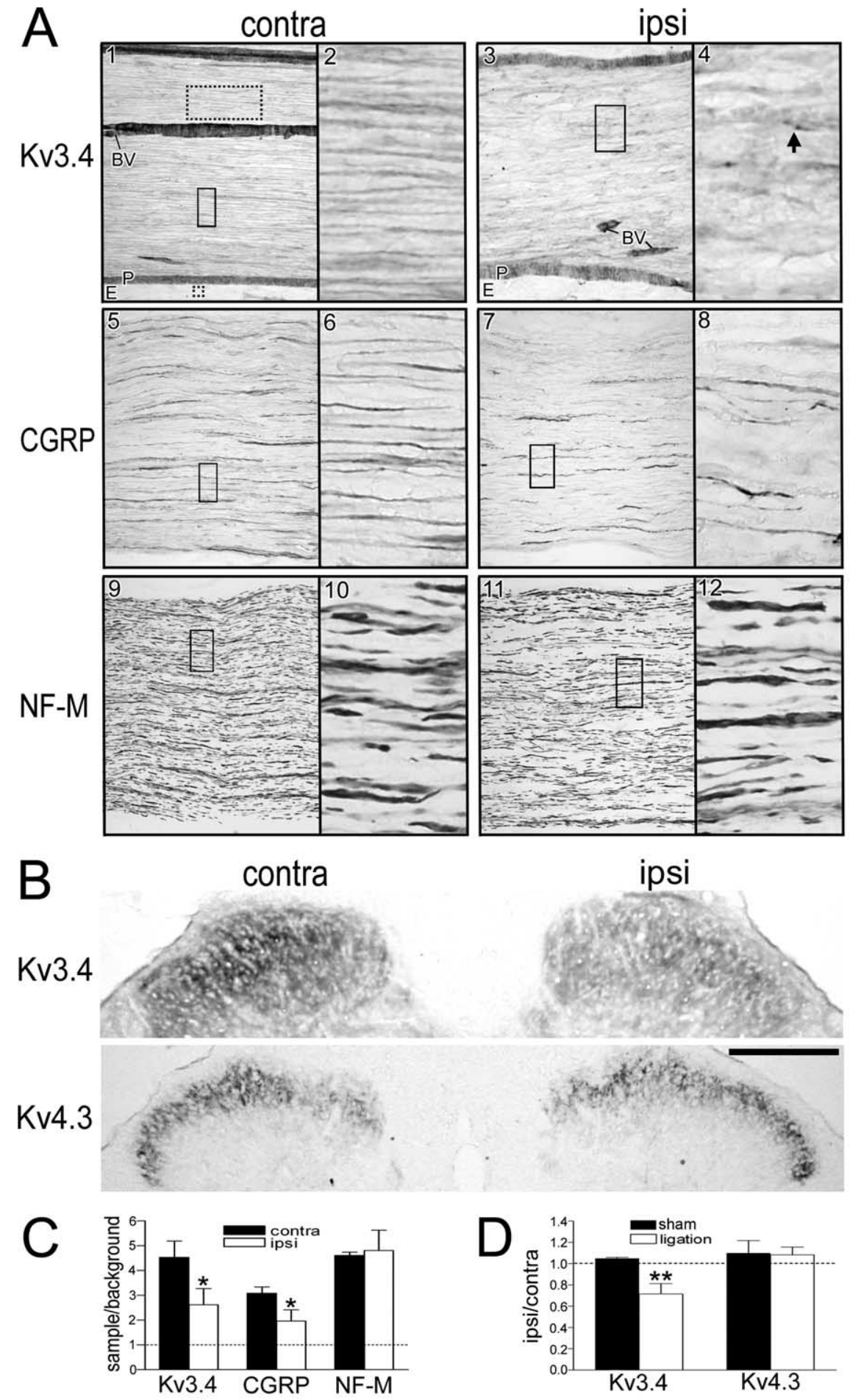

Figure 4. Reduced expression of Kv3.4 in the peripheral axons and central afferent terminals of DRG neurons after spinal nerve ligation. $\boldsymbol{A}$, Sciatic nerves from the nonligated (contra) and ligated (ipsi) sides were immunostained with antibody against Kv3.4, CGRP, or NF-M. Odd-numbered panels are at lower magnifications, and the regions within the rectangles (solid line) are magnified in the adjacent even-numbered panels. On the ligated side, sciatic nerve shows a reduced expression of Kv3.4 and CGRP but not NF-M. $\boldsymbol{B}$, In the dorsal horn of L5 spinal cord, Kv3.4-IR is weaker on the ligated side, whereas Kv4.3-IR shows no difference. $\boldsymbol{C}$, Quantification of $\boldsymbol{A}$. The first panel in $\boldsymbol{A}$ is used as a representative for illustration. Kv3.4-IR within the large rectangle (dash line) is measured as the sample value, whereas immunoreactivity within the small square (dash line) in the epineurium is measured as the background value. D, Quantification of $\boldsymbol{B}$. Kv3.4-IR in laminas I-III or Kv4.3-IR in lamina II was measured as the sample value. Immunoreactivity within five areas of the white matter (one in the dorsal funiculus as well as the other four in the lateral and bottom parts of both sides of spinal cord) were measured and averaged as the background value. $n=4 ;{ }^{*} p<0.05,{ }^{* *} p<0.01$, comparing the ligated side (white bar) with the nonligated side (black bar) by Student's t test. BV, Blood vessel; E, epineurium; $P$, perineurium. Scale bar: (in $\boldsymbol{B}) \boldsymbol{A}$, panels in odd numbers, $300 \mu \mathrm{m}$; panels in even numbers, $38 \mu \mathrm{m} ; \boldsymbol{B}, 250 \mu \mathrm{m}$. 
uptake into lumbar DRG, we made a single intrathecal injection of fluorescence-labeled Kv3.4 antisense ODNs. The fluorescence intensity in the L5 DRG neurons was strong at $5 \mathrm{~h}$, moderate at $8 \mathrm{~h}$, and weak at 3 and $12 \mathrm{~h}$ after the injection (supplemental data, available at www.jneurosci.org as supplemental material) (Fig. 4). Fluorescence-tagged Kv4.3 antisense ODNs showed a similar profile (data not shown). Behavioral tests were therefore performed 5-7 $\mathrm{h}$ after the morning injection (9:00 A.M.) (i.e., at 2:00-4:00 P.M.). The antisense ODNs (without fluorescence tag), mismatch ODNs, and vehicle were given in parallel twice daily (9:00 A.M. and 9:00 P.M.) for days 1-3. There was no injection during days $4-7$. In rats treated with Kv3.4 or Kv4.3 antisense ODNs, mechanical hypersensitivity developed on the second day, remained obvious on the third and fourth days, partially subsided on the fifth day, and completely disappeared on the sixth and seventh days (Fig. 5A). In contrast, thermal hypersensitivity was not observed (Fig. $5 B$ ). Both behavioral tests showed no significant change in rats injected with mismatch ODNs or vehicle (Fig. $5 A, B$ ).

To test whether there is a correlation between mechanical sensitivity and A-channel protein level, we measured Kv3.4 and Kv4.3-IR in the L5 DRG neurons at different days after antisense ODN treatment. Rats were killed $\sim 1-6 \mathrm{~d}$ after the first injection (i.e., at 9:00 A.M. on the second to seventh days). Kv3.4 and Kv4.3-IR were reduced $1 \mathrm{~d}$ later (Fig. $6 A$ ), and mechanical sensitivity increased significantly on the second day (Fig. $5 A$ ). This suggests that mechanical hypersensitivity develops after the decrease of A-channels. Three days after the first antisense ODN injection, Kv3.4 and Kv4.3-IR reduced to 46 and 33\% of the vehicle group, respectively; however, CGRP $(+)$ DRG neurons remained at the same expression level with intact morphology (Fig. 6B,C). Kv3.4 antisense ODNs had no effect on Kv4.3-IR and vice versa (Fig. $6 C$ ). These data indicated that DRG neurons were not damaged by consecutive $3 \mathrm{~d}$ antisense ODN treatment and that the knockdown effect was gene-specific. Furthermore, the antisense effect was reversible. Kv3.4 and Kv4.3-IR in the DRG neurons gradually returned to normal levels after stopping the injection (Fig. 6A). Consistent with this result, mechanical sensitivity retuned to normal level $2 \mathrm{~d}$ after stopping the injection (Fig. 5A). In summary, our data strongly suggest a causal role of A-channel suppression in mechanical hypersensitivity.

\section{Mechanical hypersensitivity can be induced by suppressing A-channel expression in nociceptors}

Knockout of A-channel expression in dorsal horn neurons can also lead to mechanical hypersensitivity (Hu et al., 2006). It suggests that mechanical hypersensitivity can be induced by antisense ODNs acting on DRG or dorsal horn neurons. Although both DRG and spinal cord are located in the subarachnoid space, neurons within spinal cord are further protected by the pia mater, the continuous sheath formed by subpial astrocytes, and the white matter (Parent, 1996). Because of poor uptake of antisense ODNs into spinal cord, a decrease of voltage-gated $\mathrm{Ca}^{2+}$ channel Cav3.2 mRNAs and proteins has been observed only in lumbar DRGs after intrathecal antisense ODN injection (Bourinet et al., 2005). For confirmation, we examined the uptake of fluorescence-labeled Kv3.4 and Kv4.3 antisense ODNs into L5 spinal cord, respectively. In all time points examined $(3,5,8$, and $12 \mathrm{~h}$ ) for both ODNs, except the pia mater, no significant fluorescence signal could be detected in lumbar spinal cord (data not shown). These data imply that the concentration of antisense ODNs in dorsal horn neurons would be too low to suppress gene translation. To verify this, we measured Kv3.4 and Kv4.3-IR in
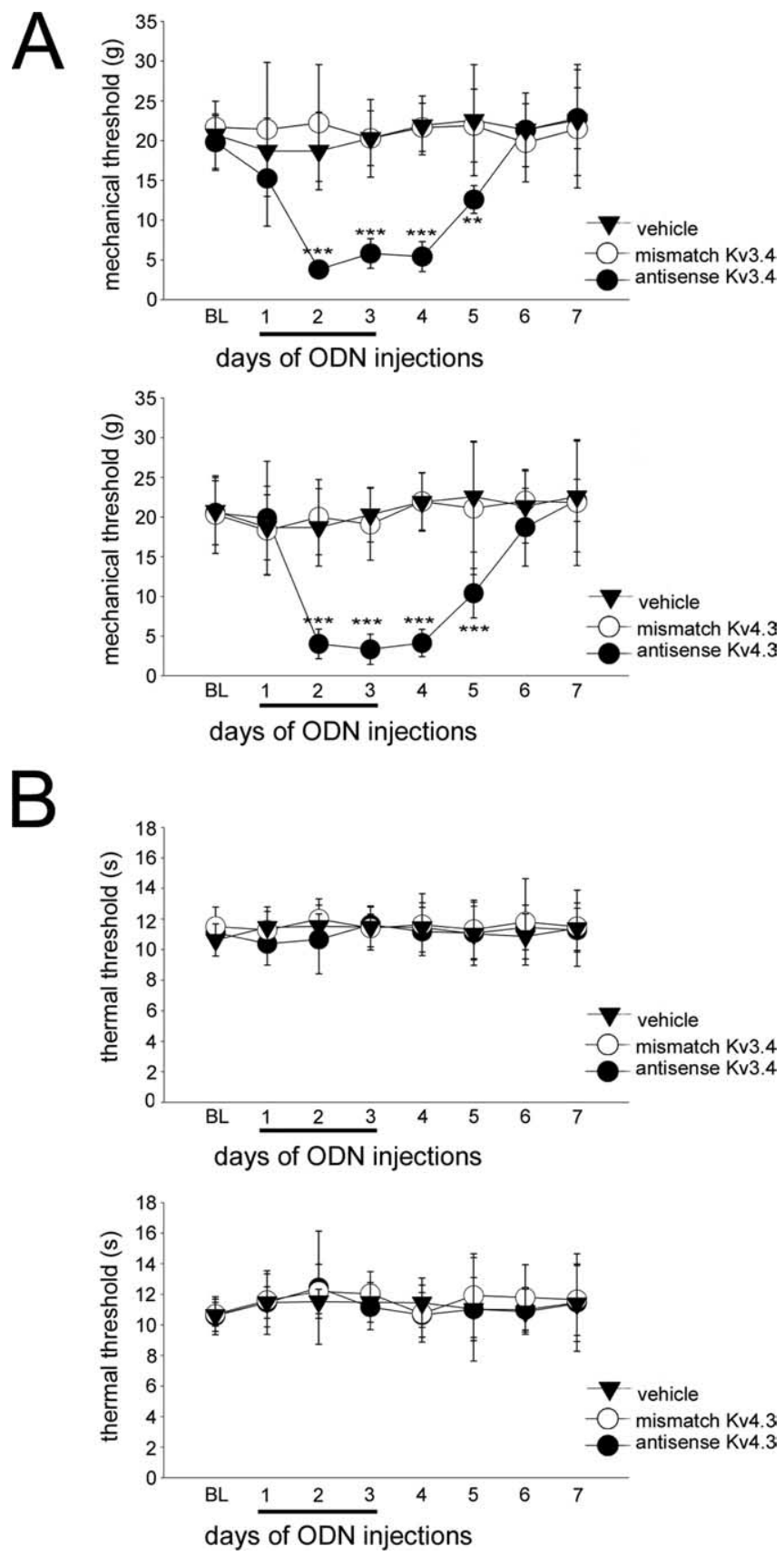

Figure 5. Mechanical hypersensitivity is induced after Kv3.4 or Kv4.3 antisense ODN treatment. Rats were intrathecally injected with vehicle, mismatch, or antisense ODNs twice daily at 9:00 A.M. and 9:00 P.M. for three consecutive days (underlined below 1, 2, and 3). Behavioral tests were performed once daily at 2:00-4:00 P.M. A, Mechanical hypersensitivity became apparent on the second day of antisense ODN injection and gradually disappeared after stopping the injection. $\boldsymbol{B}$, Thermal hypersensitivity was not detected. $n=6$; ${ }^{* *} p<0.01,{ }^{* * *} p<$ 0.001 , comparing the response of each day with baseline (BL; $1 \mathrm{~d}$ before the first injection) by Student's $t$ test.

the L5 dorsal horn from rats injected with vehicle, mismatch or antisense ODNs for $3 \mathrm{~d}$. Kv3.4-IR was reduced to $62 \%$ by Kv3.4 antisense ODNs; however, Kv4.3-IR showed no significant change by Kv4.3 antisense ODNs (Fig. 7A,B). Decrease of Kv3.4-IR in the dorsal horn was contributed mainly by reduced Kv3.4 expression in DRG neurons, whereas no alteration of $\mathrm{Kv} 4.3$-IR in the dorsal horn indicated a normal Kv4.3 expression in dorsal horn neurons. Our data suggest that the concentration of antisense ODNs used here is high enough to exert a suppress- 
ing effect in DRG but not dorsal horn neurons. It is also suggested that mechanical hypersensitivity can be induced solely by suppressing A-channel expression in primary sensory neurons.

\section{Discussion}

Four major findings arise from this study. First, Kv3.4 is expressed mainly in the C-fiber nociceptors, in their somata, axons, and nerve terminals (Fig. $8 A$ ). Second, Kv4.3 is expressed selectively in the somata of a subset of C-fiber nociceptors (Fig. 8 A). Most Kv4.3(+) nociceptors also express Kv3.4. Third, after neuropathic pain was induced by nerve ligation, both mechanical and thermal hypersensitivities were observed and the expression levels of Kv3.4 and Kv4.3 in the nociceptors were greatly reduced (Fig. $8 \mathrm{~B}$ ). Fourth, after the expression level of Kv3.4 or Kv4.3 in the nociceptors were suppressed by antisense ODN treatment (Fig. 8C), mechanical hypersensitivity was selectively induced.

\section{Selective expression of $\mathrm{Kv} 4.3$ in a subset of nociceptors supports the labeled lines theory}

The "labeled lines theory" illustrates that there are different pain pathways and each pathway has its own anatomical structures to carry restricted information of pain (Craig, 2003; Braz et al., 2005). For example, using IB4 and CGRP as markers, nociceptive DRG neurons and their projection fields in the dorsal horn of spinal cord have been divided into two distinct anatomical pathways (Hunt and Mantyh, 2001; Julius and Basbaum, 2001). Recently, Zylka et al. (2005) showed Mrgprd (a sensory neuron-specific G-proteincoupled receptor) in a subset of $\operatorname{IB} 4(+)$ DRG neurons projecting exclusively to skin, again supporting this theory. Here, we observed $\mathrm{Kv} 4.3$ in a subset of IB4 $(+)$ DRG neurons, similar to $\operatorname{Mrgprd}(+)$ DRG neurons. All Kv4.3(+) neurons were IB4 $(+)$ and $44 \%$ of IB4 $(+)$ neurons expressed Kv4.3. Coincidently, all Mrg$\operatorname{prd}(+)$ neurons were IB4 $(+)$ and $48 \%$ of IB4 $(+)$ neurons expressed Mrgprd (Zylka et al., 2005). In addition, Kv4.3 was present in $50 \%$ of $\mathrm{VR} 1(+)$ neurons, and Mrgprd was found in $51 \%$ of $\operatorname{VR} 1(+)$ neurons (Zylka et al., 2005). These data imply that Kv4.3 and Mrgprd are coexpressed in the same subset of IB4(+) DRG neurons selectively innervating skin. Together with the finding that mechanical hypersensitivity developed after Kv4.3 downregulation, our data suggest that Kv4.3(+) DRG neurons could be the anatomical structure transmitting information of mechanical stimuli from skin. Although more experimental evidence will be required, selective expression of $\mathrm{Kv} 4.3$ in a subset of IB4 $(+)$ nociceptors supports the label lines theory.

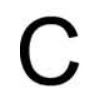

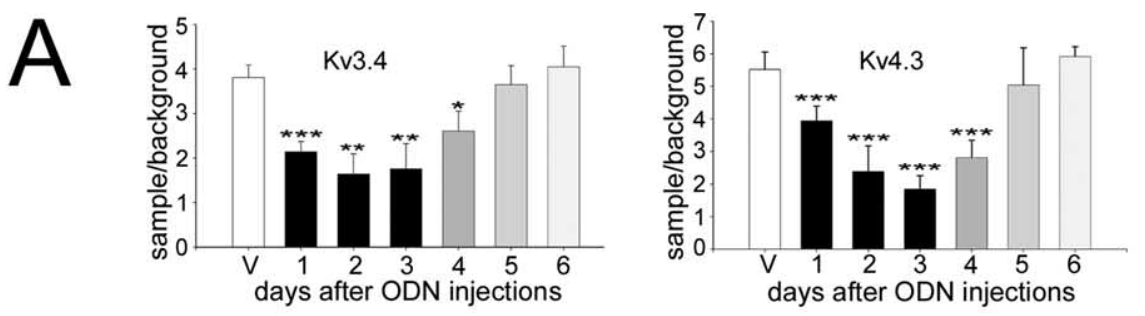

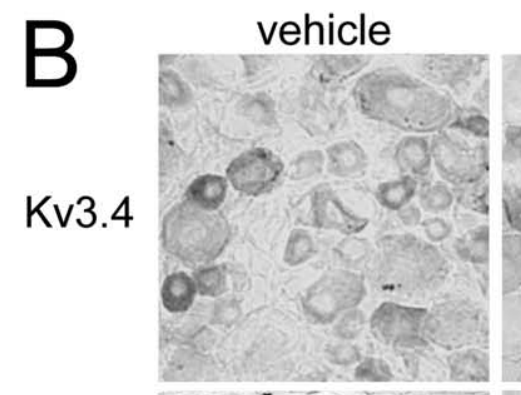

\section{mismatch}

antisense
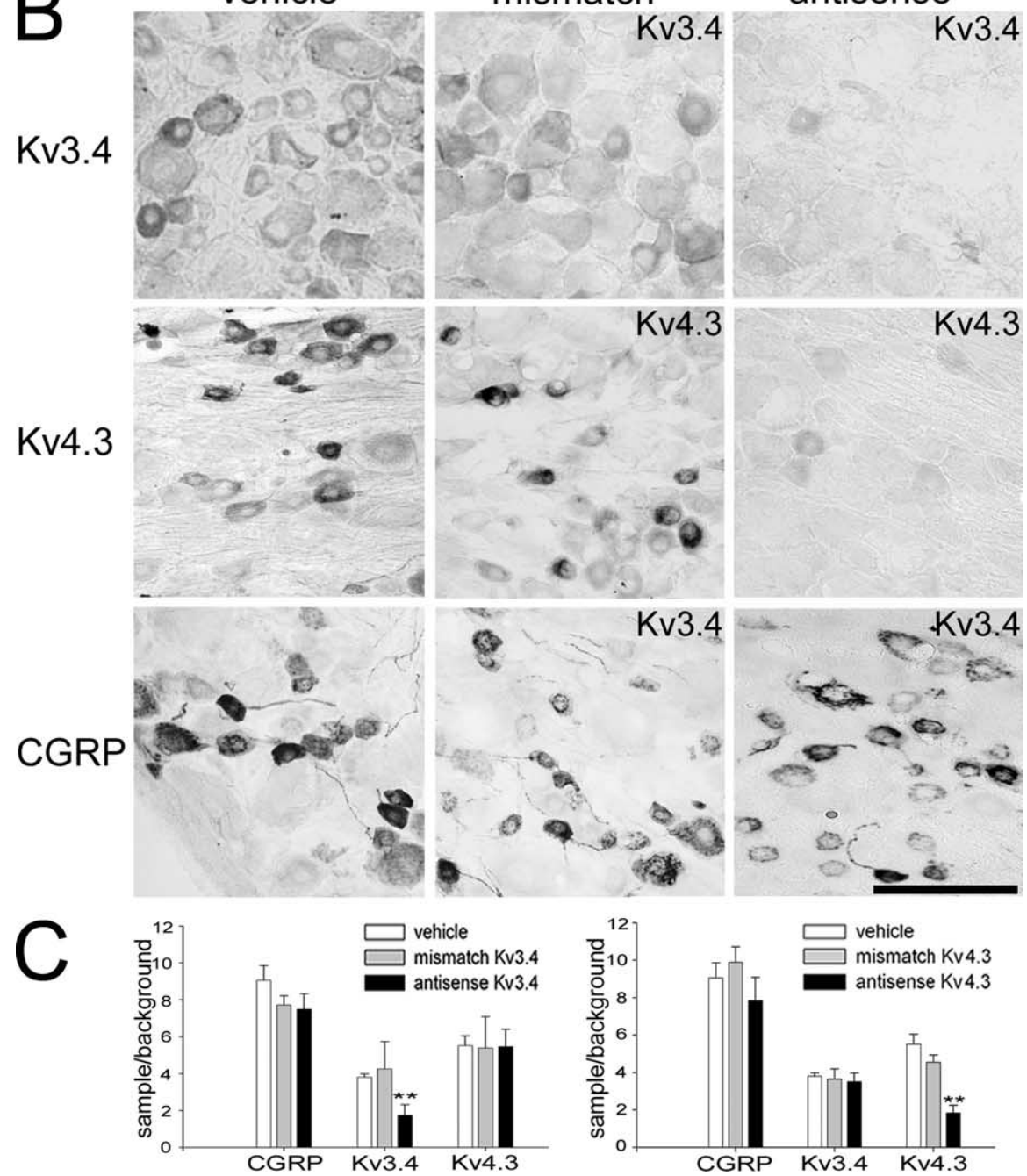

Figure 6. Reduced expression of Kv3.4 and Kv4.3 in the DRG neurons by gene-specific antisense ODNs. Rats were intrathecally injected with vehicle, mismatch, or antisense ODNs for three consecutive days. Sections of L5 DRG were immunostained with antibody against Kv3.4, Kv4.3, or CGRP. A, In the rats treated with gene-specific antisense ODNs, Kv3.4 and Kv4.3-IR in the DRG neurons decreased $1 \mathrm{~d}$ later, remained at low levels during consecutive injections, and gradually reversed to normal levels after stopping the injection on the fourth day. $\boldsymbol{B}$, After injections for three consecutive days, Kv3.4 and Kv4.3-IR were reduced by gene-specific antisense ODNs but not by mismatch ODNs or vehicle. CGRP-IR was not altered by Kv3.4 or Kv4.3 mismatch and antisense ODNs (only data of Kv3.4 is shown). Scale bar, $116 \mu \mathrm{m}$. C, Quantification of immunoreactivity in $B . n \geq 4$; ${ }^{*} p<0.05$, ${ }^{* *} p<0.01,{ }^{* *} p<0.001$, comparing the antisense or mismatch with vehicle group (V) by Student's $t$ test.

\section{Coexpression of distinct A-channels enables a nociceptor to reflect different intensities of stimuli}

We detected Kv4.3 in 26\% of DRG neurons that are exclusively IB4 $(+)$ and also found Kv3.4 in 79\% of Kv4.3(+) DRG neurons. That is, $21 \%(26 \times 79 \%)$ of DRG neurons express both Kv3.4 and Kv4.3. In addition, all IB4(+) DRG neurons express Kv1.4 (Vydyanathan et al., 2005). These data suggest that $21 \%$ of DRG neurons coexpress Kv1.4, Kv3.4, and Kv4.3. In the heterologous 


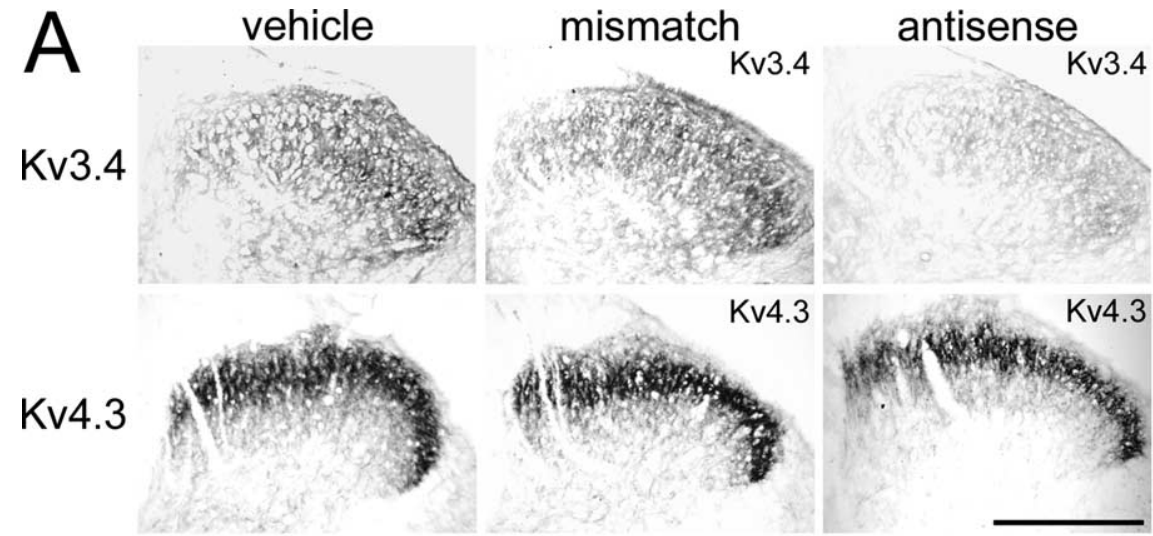

B
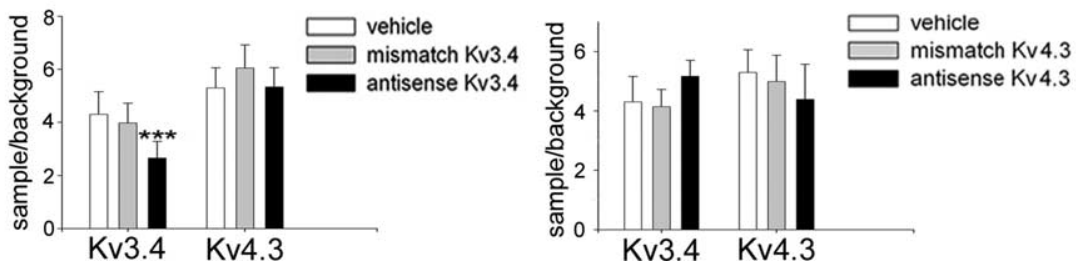

Figure 7. Reduced expression of Kv3.4 but not Kv4.3 in the dorsal horn by gene-specific antisense 0DNs. Rats were intrathecally injected with vehicle, mismatch, or antisense ODNs for three consecutive days. Sections of $L 5$ spinal cord were immunostained with antibody against Kv3.4 or Kv4.3. $\boldsymbol{A}$, In the dorsal horn (only the right side is shown), Kv3.4-IR was reduced by Kv3.4 antisense ODNs, whereas Kv4.3-IR was not affected by Kv4.3 antisense ODNs. Scale bar, $300 \mu \mathrm{m}$. $\boldsymbol{B}$, Quantification of $\boldsymbol{A}$. Immunoreactivity in each side of the spinal cord was measured, and an average value was obtained from both sides. $n=6$; ${ }^{* * *} p<0.001$, comparing the antisense or mismatch with vehicle group by Student's $t$ test.

expression systems, these A-channels differ in the activation threshold: subthreshold for Kv4.3 (approximately -50 to -60 $\mathrm{mV}$ ), low-threshold for Kv1.4 (approximately -40 to $-50 \mathrm{mV}$ ), and high-threshold for Kv3.4 (at least $-10 \mathrm{mV}$ ) (Coetzee et al., 1999). Because pain is a graded sensory response, we hypothesize that coexpression of three distinct A-channels may enable a painsensing neuron to reflect different intensities of stimuli in the following manner. When a weak stimulus slightly depolarizes membrane potential to approximately -50 to $-60 \mathrm{mV}, \mathrm{Kv} 4.3$ channels can open to dampen the signal. When a moderate stimulus depolarizes the membrane potential to approximately -40 to $-50 \mathrm{mV}$, because both $\mathrm{Kv} 1.4$ and $\mathrm{Kv} 4.3$ channels are activated below the action potential threshold, they can act together to prevent action potentials from being evoked. When a stimulus is sufficiently strong to evoke action potentials (less than $-40 \mathrm{mV}$ ), the strength of the stimulus is encoded by the spiking frequency. During neuronal firing at high frequency, opening of Kv3.4 channels can rapidly repolarize the membrane potential to ensure quick recovery of voltage-gated $\mathrm{Na}^{+}$channels from inactivation (Rudy and McBain, 2001). Keeping in this line, various combinations of A-channels in different subsets of primary sensory neurons can therefore provide diverse responses to external stimuli. It is worth noting that the activation threshold of A-channel could be changed after forming heteromultimers with other proteins. Two examples are given as follows. Kv3.4 and delayed rectifier $\mathrm{K}^{+}$channel Kv3.1 or Kv3.2 form heterotetramers in fastspiking neurons and the resulting fast delayed rectifier $\mathrm{K}^{+}$ currents are activated at $-30 \mathrm{mV}$ (Baranauskas et al., 2003). Kv4.3 and KChIP1 form heteromultimers in the hippocampal interneurons and the resulting $I_{\mathrm{A}} \mathrm{s}$ can be activated at more hyperpolarized potentials (An et al., 2000; Rhodes et al., 2004)). Whether A-channels form heteromultimers with other poreforming or auxiliary subunits in pain-sensing neurons remains to be elucidated.

\section{Relationship between A-channels and nerve ligation-induced neuropathic pain}

We have shown a reduction of Kv3.4 and Kv4.3 proteins in the nociceptive DRG neurons after spinal nerve ligation. Together with Kv1.4 (Rasband et al., 2001), it appears that A-channel expression level in the nociceptors and nerve ligationinduced neuropathic pain are closely related. Using antisense ODNs to suppress $\mathrm{Kv} 3.4$ or Kv4.3 expression in the nociceptors, we found animals developing mechanical hypersensitivity, a major symptom of neuropathic pain. Our data suggest that the A-channel protein level in nociceptors decreases before the induction of mechanical hypersensitivity. Reduced A-channels on the cell surface make nociceptors hyper-excitable and cannot precisely reflect the intensity of a stimulus. This would convert a normally innocuous tactile stimulus turns into a noxious stimulus. In addition to reduced expression, $I_{\mathrm{A}} \mathrm{s}$ can also be suppressed if the channel becomes phosphorylated (Beck et al., 1998; Birnbaum et al., 2004; Sergeant et al., 2005). Phosphorylation of Kv4.2 in dorsal horn neurons has been proposed to underlie the induction of central sensitization, a cellular mechanism of neuropathic pain (Ji et al., 2003). Using dorsal horn neurons isolated from Kv4.2 knock-out mice, $\mathrm{Hu}$ et al. (2006) have shown that Kv4.2 phosphorylation by extracellular signal-regulated kinase is critical for pain modulation. Although the total amount of Kv4.3 proteins in dorsal horn neurons showed no significant decrease after spinal nerve ligation, whether the percentage of phosphorylated Kv4.3 proteins increases requires additional investigation.

\section{Molecules for selective induction of mechanical hypersensitivity}

Cumulating evidence indicates that mechanical and thermal hypersensitivities, two major symptoms of neuropathic pain, can be dissociated (Ossipov et al., 2002; Obata et al., 2004; Soliman et al., 2005; Son et al., 2007). After chronic constriction injury of sciatic nerve, the transcription factor c-Jun becomes highly expressed in DRG neurons, and intrathecal c-Jun antisense ODN treatment can reduce mechanical but not thermal hypersensitivity (Son et al., 2007). Similarly, after spinal nerve ligation, phosphorylated c-Jun N-terminal kinase (pJNK) in small-sized DRG neurons greatly increases and intrathecal infusion of pJNK inhibitor can reverse mechanical but not thermal hypersensitivity (Obata et al., 2004; Zhuang et al., 2006). In this study, we found that A-channels Kv3.4 and Kv4.3 are abundantly expressed in the nociceptive DRG neurons. When their protein levels are suppressed by antisense ODN treatment, again, mechanical but not thermal hypersensitivity is induced. These studies indicate that c-Jun, pJNK, and A-channels are molecules related more closely to mechanical than thermal sensitivity. It will be interesting to investigate the relationship between these molecules in the induction of mechanical hypersensitivity. 


\section{A DRG}

Nociceptive sensory neurons

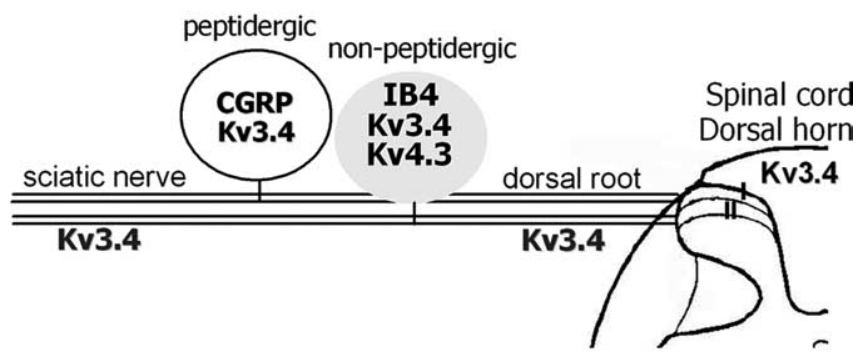

B

DRG

Nociceptive sensory neurons
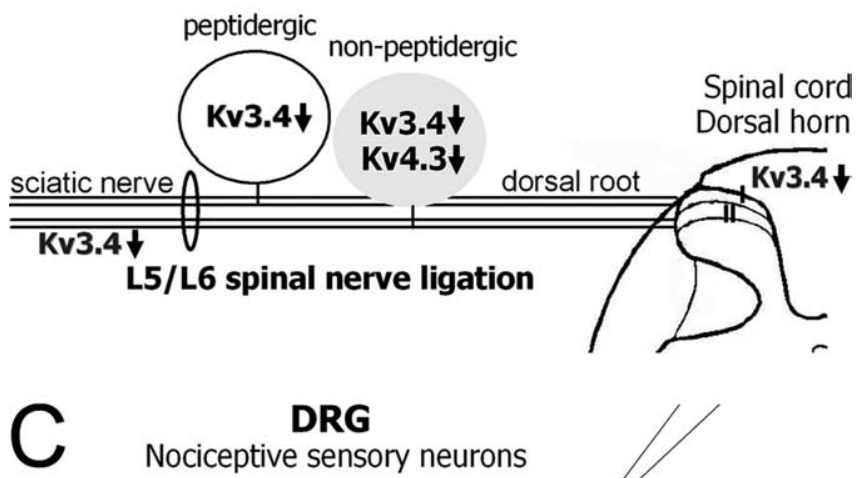

DRG

Nociceptive sensory neurons
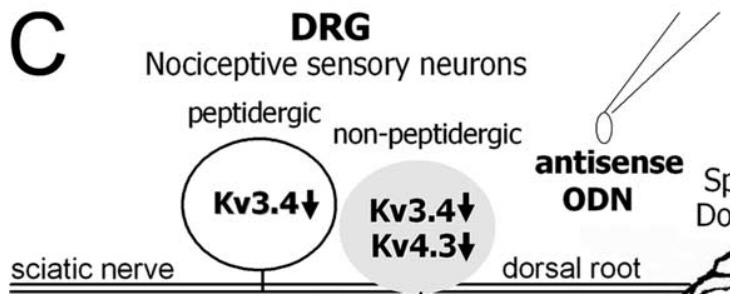

ODN

Spinal cord Dorsal horn

dorsal root
Kv3.4t

members (e.g., retigabine) have been found to be antinociceptive in several animal pain models (Ocana et al., 2004), and a new compound, KW-7158, acts like an A-type $\mathrm{K}^{+}$channel opener in the DRG neurons (Sculptoreanu et al., 2004). Whether these compounds can be clinically used as analgesics is currently being investigated.

\section{References}

An WF, Bowlby MR, Betty M, Cao J, Ling HP, Mendoza G, Hinson JW, Mattsson KI, Strassle BW, Trimmer JS, Rhodes KJ (2000) Modulation of A-type potassium channels by a family of calcium sensors. Nature 403:553-556.

Baranauskas G, Tkatch T, Nagata K, Yeh JZ, Surmeier DJ (2003) Kv3.4 subunits enhance the repolarizing efficiency of Kv3.1 channels in fast-spiking neurons. Nat Neurosci 6:258-266.

Bear MF, Connors BW, Paradiso MA (2007) Neuroscience: exploring the brain. Baltimore: Lippincott Williams and Wilkins.

Beck EJ, Sorensen RG, Slater SJ, Covarrubias M (1998) Interactions between multiple phosphorylation sites in the inactivation particle of a $\mathrm{K}^{+}$channel. J Gen Physiol 112:71-84.

Birnbaum SG, Varga AW, Yuan LL, Anderson AE, Sweatt JD, Schrader LA (2004) Structure and function of Kv4-family transient potassium channels. Physiol Rev 84:803-833.

Bourinet E, Alloui A, Monteil A, Barrere C, Couette B, Poirot O, Pages A, McRory J, Snutch TP, Eschalier A, Nargeot J (2005) Silencing of the $\mathrm{Ca}_{\mathrm{v}} 3.2 \mathrm{~T}$-type calcium channel gene in sensory neurons demonstrates its major role in nociception. EMBO J 24:315-324.

Braz JM, Nassar MA, Wood JN, Basbaum AI (2005) Parallel "pain" pathway arise from subpopulations of primary afferent nociceptor. Neuron 47:787-793.

Brooke RE, Atkinson L, Batten TFC, Deuchars SA, Deuchars J (2004) Association of potassium channel Kv3.4 subunits with pre- and post-synaptic structures in brainstem and spinal cord. Neuroscience 126:1001-1010.

Burkhalter A, Gonchar Y, Mellor RL, Nerbonne JM (2006) Differential expression of $I_{\mathrm{A}}$ channel subunits Kv4.2 and Kv4.3 in mouse visual cortical neurons and synapses. J Neurosci 26:12274-12282.

Campana WM, Myers RR (2003) Exogenous erythropoietin protects against dorsal root ganglion apoptosis and pain following peripheral nerve injury. Eur J Neurosci 18:1497-1506.

Carr PA, Yamamoto T, Karmy G, Baimbridge KG, Nagy JI (1989) Parvalbumin is highly colocalized with calbindin D28K and rarely with calcitonin gene-related peptide in dorsal root ganglia neurons of rat. Brain Res 497:163-170.

Figure 8. Correlation between A-channel expression level in pain-sensing neurons and neuropathic pain. $A$, A-channels are highly expressed in pain-sensing neurons under normal condition. Kv3.4 is expressed strongly in the nonpeptidergic (IB4-positive) and weakly in the peptidergic (CGRP-positive) nociceptive DRG neurons, in the somata, peripheral axons (sciatic nerve), central axons (dorsal root), and nerve terminals innervating the superficial dorsal horn of spinal cord. In contrast, Kv4.3 appears only in the somata of nonpeptidergic DRG neurons. $\boldsymbol{B}$, Downregulation of A-channel in pain-sensing neurons may contribute to the development of nerve ligation-induced neuropathic pain. Seven days after $L / L 6$ spinal nerve ligation in rats, mechanical and thermal hypersensitivities (two major symptoms of neuropathic pain) are observed. Both Kv3.4 and Kv4.3 proteins are greatly reduced in all of the subcellular domains of L5/L6 DRG neurons normally expressing them. C, Downregulation of A-channel in pain-sensing neurons selectively induces mechanical hypersensitivity. After Kv3.4 or Kv4.3 expression in lumbar DRG neurons are suppressed by gene-specific antisense ODNs via intrathecal injections, mechanical but not thermal hypersensitivity develops.

\section{Concluding remarks}

Pain is a physiological warning system and can be switched on only by intense stimuli. However, after injury, pain could be initiated by a normally innocuous stimulus. $\mathrm{K}^{+}$channels are key components controlling neuronal excitability. Downregulation of $\mathrm{K}^{+}$channels results in neuronal hyperexcitability, which has been implicated in the development of pain. Four major types of $\mathrm{K}^{+}$channels are involved in pain modulation, including the inward-rectifier (such as ATP-sensitive and G-protein-coupled $\mathrm{K}^{+}$channels), $\mathrm{Ca}^{2+}$-activated, two-pore, and $\mathrm{Kv}$ channels. Among the Kv subunits, openers of Kv7 (KCNQ) subfamily

Chaplan SR, Bach FW, Pogrel JW, Chung JM, Yaksh TL (1994) Quantitative assessment of tactile allodynia in the rat paw. J Neurosci Methods 53:55-63.

Coetzee WA, Amarillo Y, Chiu J, Chow A, Lau D, McCormack T, Moreno H, Nadal M. S, Ozaita A, Pountney D, Saganich M, Vega-Saenz de Miera E, Rudy B (1999) Molecular diversity of $\mathrm{K}^{+}$channels. Ann NY Acad Sci 868:233-285.

Costigan M, Scholz J, Samad T, Woolf CJ (2006) Pain. In: Basic neurochemistry: molecular, cellular, and medical aspects (Seigel JG, Albers RW, Brandy ST, Price DL, eds), pp 927-937. London: Elsevier.

Craig AD (2003) Pain mechanisms: labeled lines versus convergence in central processing. Annu Rev Neurosci 26:1-30.

Gold MS, Shuster MJ, Levine JD (1996) Characterization of six voltagegated $\mathrm{K}^{+}$currents in adult rat sensory neurons. J Neurophysiol 75:2629-2646.

Gold MS, Weinreich D, Kim CS, Wang R, Treanor J, Porreca F, Lai J (2003) Redistribution of Nav1.8 in uninjured axons enables neuropathic pain. J Neurosci 23:158-166.

Hargreaves K, Dubner R, Brown F, Flores C, Joris J (1988) A new and sensitive method for measuring thermal nociception in cutaneous hyperalgesia. Pain 32:77-88.

Hasdemir B, Fitzgerald DJ, Prior IA, Tepikin AV, Burgoyne RD (2005) Traffic of $\mathrm{Kv} 4 \mathrm{~K}^{+}$channels mediated by KChIP1 is via a novel post-ER vesicular pathway. J Cell Biol 171:459-469.

Hille B (2001) Ionic channels of excitable membranes. Sunderland, MA: Sinauer.

Hsu YH, Huang HY, Tsaur ML (2003) Contrasting expression of Kv4.3, an A-type $\mathrm{K}^{+}$channel, in migrating Purkinje cells and other post-migratory cerebellar neurons. Eur J Neurosci 18:601-612. 
Hu HJ, Carrasquillo Y, Karim F, Jung WE, Nerbonne JM, Schwarz TL, Gereau RW (2006) The Kv4.2 potassium channel subunit is required for pain plasticity. Neuron 50:89-100.

Huang HY, Cheng JK, Shih YS, Chen PS, Wang CL, Tsaur ML (2005) Expression of A-type $\mathrm{K}^{+}$channel $\alpha$ subunits $\mathrm{Kv} 4.2$ and $\mathrm{Kv} 4.3$ in rat spinal lamina II excitatory interneurons and co-localization with painmodulating molecules. Eur J Neurosci 22:1149-1157.

Huang HY, Liao CW, Chen PS, Tsaur ML (2006) Transient expression of A-type $\mathrm{K}^{+}$channel $\alpha$ subunits $\mathrm{Kv} 4.2$ and $\mathrm{Kv} 4.3$ in rat spinal neurons during development. Eur J Neurosci 23:1142-1150.

Hunt SP, Mantyh PW (2001) The molecular dynamics of pain control. Nature Rev 2:83-91.

Ji RR, Kohno T, Moore KA, Woolf CJ (2003) Central sensitization and LTP: do pain and memory share similar mechanisms? Trends Neurosci 26:696-705.

Julius D, Basbaum AI (2001) Molecular mechanisms of nociception. Nature 413:203-210.

Kim SH, Chung JM (1992) An experimental model for peripheral neuropathy produced by segmental spinal nerve ligation in the rat. Pain 50:355-363.

Lai J, Gold MS, Kim CS, Bian D, Ossipov MH, Hunter JC, Porreca F (2002) Inhibition of neuropathic pain by decreased expression of the tetrodotoxin-resistant sodium channel, NaV1.8. Pain 95:143-152.

Obata K, Yamanaka H, Kobayashi K, Dai Y, Mizushima T, Katsura H, Fukuoka T, Tokunaga A, Noguchi K (2004) Role of mitogen-activated protein kinase activation in injured and intact primary afferent neurons for mechanical and heat hypersensitivity after spinal nerve ligation. J Neurosci 24:10211-10222.

O'Callaghan DW, Hasdemir B, Leighton M, Burgoyne RD (2003) Residues within the myristoylation motif determine intracellular targeting of the neuronal $\mathrm{Ca}^{2+}$ sensor protein KChIP1 to post-ER transport vesicles and traffic of Kv4 K ${ }^{+}$channels. J Cell Sci 116:4833-4845.

Ocana M, Cendan CM, Cobos EJ, Entrena JM, Baeyens JM (2004) Potassium channels and pain: present realities and future opportunities. Eur J Pharmacol 500:203-219.

Ossipov MH, Zhang ET, Carvajal C, Gardell L, Quirion R, Dumont Y, Lai J, Porreca F (2002) Selective mediation of nerve-injury induced tactile hypersensitivity by neuropeptide Y. J Neurosci 22:9858-9867.

Parent A (1996) Carpenter's human neuroanatomy. Baltimore: William and Wilkins.

Rasband MN, Park EW, Vanderah TW, Lai J, Porreca F, Trimmer JS (2001) Distinct potassium channels on pain-sensing neurons. Proc Natl Acad Sci USA 23:13373-13378.

Rhodes KJ, Carroll KI, Sung MA, Doliveira LC, Monaghan MM, Burke SL, Strassle BW, Buchwalder L, Menegola M, Cao J, An WF, Trimmer JS (2004) KChIPs and Kv4 $\alpha$ subunits as integral components of A-type potassium channels in mammalian brain. J Neurosci 24:7903-7915.

Rice ASC, Hill RG (2006) New treatments for neuropathic pain. Annu Rev Med 57:535-551.
Rudy B, McBain CJ (2001) Kv3 channels: voltage-gated $\mathrm{K}^{+}$channels designed for high-frequency repetitive firing. Trends Neurosci 24:517-526.

Schroter KH, Ruppersberg JP, Wunder F, Rettig J, Stocker M, Pongs O (1991) Cloning and functional expression of a TEA-sensitive A-type potassium channel from rat brain. FEBS Lett 278:211-216.

Sculptoreanu A, Yoshimura N, de Groat WC (2004) KW-7158 [(2S)-(+)3,3,3-Trifluoro-2-hydroxy-2-methyl-N-(5,5,10-trioxo-4,10dihydrothieno[3,2-c][1]benzothiepin-9-yl)propanamide] enhances A-type $\mathrm{K}^{+}$currents in neurons of the dorsal root ganglion of the adult rat. J Pharmacol Exp Ther 310:159-168.

Sergeant GP, Ohya S, Reihill JA, Perrino BA, Amberg GC, Imaizumi Y, Horowitz B, Sanders KM, Koh SD (2005) Regulation of Kv4.3 currents by $\mathrm{Ca}^{2+} /$ calmodulin-dependent protein kinase II. Am J Physiol Cell Physiol 288:C304-C313.

Shindler KS, Roth KA (1996) Double immunofluorescent staining using two unconjugated primary antisera raised in the same species. J Histochem Cytochem 44:1331-1335.

Soliman AC, Yu JSC, Coderre TJ (2005) mGlu and NMDA receptor contributions to capsicin-induced thermal and mechanical hypersensitivity. Neuropharmacol 48:325-332.

Son SJ, Lee KM, Jeon SM, Park ES, Park KM, Cho HJ (2007) Activation of transcription factor $c$-jun in dorsal root ganglia induced VIP and NPY upregulation and contributes to the pathogenesis of neuropathic pain. Exp Neurol 204:467-472.

Tandrup T, Woolf CJ, Coggeshall RE (2000) Delayed loss of small dorsal root ganglion cells after transaction of the rat sciatic nerve. J Comp Neurol 422:172-180.

Tsaur ML, Chou CC, Shih YH, Wang HL (1997) Cloning, expression and CNS distribution of $\mathrm{Kv} 4.3$, an A-type $\mathrm{K}^{+}$channel $\alpha$ subunit. FEBS Lett 400:215-220.

Vydyanathan A, Wu ZZ, Chen SR, Pan HL (2005) A-type voltage-gated $\mathrm{K}^{+}$currents influence firing properties of isolectin $\mathrm{B}_{4}$-positive but not isolectin $\mathrm{B}_{4}$-negative primary sensory neurons. J Neurophysiol 93:3401-3409.

Yaksh TL, Rudy TA (1976) Chronic catheterization of the spinal subarachnoid space. Physiol Behav 17:1031-1036.

Zhuang ZY, Wen YR, Zhang DR, Borsello T, Bonny C, Strichartz GR, Decosterd I, Ji RR (2006) A peptide c-Jun N-terminal kinase (JNK) inhibitors blocks mechanical allodynia after spinal nerve ligation: respective roles of JNK activation in primary sensory neurons and spinal astrocytes for neuropathic pain development and maintenance. J Neurosci 26:3551-3560.

Zwick M, Davis BM, Woodbury CJ, Burkett JN, Koerber HR, Simpson JF, Albers KM (2002) Glial cell line-derived neurotrophic factor is a survival factor for isolectin B4-positive, but not vallinoid receptor 1-positive, neurons in the mouse. J Neurosci 22:4057-4065.

Zylka MJ, Rice FL, Anderson DJ (2005) Topographically distinct epidermal nociceptive circuits revealed by axonal tracers targeted to Mrgprd. Neuron 45:17-25. 